\title{
II. On the refraction of rays of great wave-length in rock-salt, sylvite, and fluorite
}

\section{Heinrich Rubens \& Benj. W. Snow}

To cite this article: Heinrich Rubens \& Benj. W. Snow (1893) II. On the refraction of rays of great wave-length in rock-salt, sylvite, and fluorite , Philosophical Magazine Series 5, 35:212, 35-45, DOI: $10.1080 / 14786449308620376$

To link to this article: http://dx.doi.org/10.1080/14786449308620376

曲 Published online: 08 May 2009.

Submit your article to this journal $\sqsubset$

Џ Article views: 5

Q View related articles $₫$ 
nitrogen is formed. This compound is probably identical with the nitride obtained by Plantamour.

4. The rate of formation of this nitride, other things being the same, is so irregular as to suggest that its formation is due to the interaction of an otherwise inappreciable trace of some other substance.

5. This compound is dissociable ; but under the circumstances of the experiment the dissociation process is not entirely reversible, since sufficient heating will permanently destroy the compound.

6. There are changes in the appearance of the discharge in nitrogen which can be brought about by very slight variations of temperature, and possibly of electromotive force, for which, so far as I know, no explanation is at present fortheoming.

Sydney, August 5, 1892.

II. On the Refraction of Rays of Great Wave-length in Rock-salt, Syluite, and Fluorite. By HeInRICH Rubens and BENJ. W. SNow*

\section{[Plate II.]}

N volume xl. of Wiedemann's Annalen one of the present 1 authors recently described a method whereby a knowledge of the dispersion of rays in the infra-red may be easily obtained. With the aid of this device the dependence of the index of refraction upon the wave-length was determined for sixteen materials; viz. for nine different samples of glass, for water, carbon disulphide, xylol, benzol, quartz, rock-salt, and fluorite. Inasmuch as in this paper is given a minute description of the methods employed, it will suffice here briefly to refer to the main features of the method of procedure followed in the present determination.

The rays from the zirconia burner of Linnemann, after being reflected from the front and the rear surfaces of a thin plate of air, enclosed between two parallel glass planes, were then concentrated upon the slit of a spectrometer, by which means two beams of light were produced, capable of mutual interference, so that the otherwise continuous spectrum of the incandescent zirconia plate was crossed by a series of vertical interference-bands. The wave-length $\lambda$ of each such dark band, multiplied by a certain whole number $m$, always equals the product of twice the thickness $d$ of the layer of air and the cosine of the angle of incidence $i$ of the rays. With the aid

* Communicated by the Authors.

$\mathrm{D} 2$ 
of the Fraunhofer lines the wave-]engths of the interferencebands were determined for the visible portion of the spectrum, and from these data were calculated the order $m$ of each dark band and the product $\mathrm{K}=2 d \cos i$. The knowledge of these two constants proved, then, sufficient to determine also the wave-lengths of the interference-bands in the infra-red.

The positions of these latter were obtained by allowing the sensitive filament of a linear bolometer to wander through the spectrum, and plotting the observed galvanometer-deflexions as a function of the angular deviation. The interferencebands are then recognized as minima oi maxima in the curve. In this way, for a series of angular deviations may be determined the corresponding indices of refraction; that is, a number of points in the $n-\lambda$ plane determined, which, when joined by a smooth curve, give the curve of dispersion for the material examined.

In the majority of the bodies thus investigated, the limit of the region in the infra-red capable of being explored was prescribed by the absorption, which increases rapidly with increasing wave-lengths. In two cases alone, viz. when working with rock-salt and with fluorite, were the investigations discontinued at wave-lengths $\lambda=5 \cdot 7 \mu$ and $\lambda=3 \cdot 3 \mu$ respectively, before the region of strong absorption was reached. This was rendered necessary by the fact that the apparatus employed proved to be insufficiently sensitive to measure the exceedingly feeble energy found in the spectrum of the zirconia burner at these long wave-lengths.

As a means of continuing the investigation beyond this point two ways of improvement suggested themselves. At first we thought it possible to increase the energy of the source of light, but all endeavours to attain this end proved of no avail. The use of the electric arc for this purpose was, after a short but thorough trial, discontinued. Even are lamps of unusually good regulation, when supplied by the almost perfectly constant current of the Berlin Central Station, gave a radiation too fluctuating to be used in place of the zirconia light. The regulation of the arc by hand was also tried, but also without success. The use, moreover, of a zirconia burner of nearly double the dimensions of the former resulted in only a feeble increase in the energy, while a series of new difficulties was thereby introduced, such as the melting of the platinum cell, a greater consumption of gas, \&e. We concluded, therefore, for the further investigation, to retain the source of light in its original form, and to make better use of the energy here at hand by increasing as far as possible the sensitiveness of the measuring apparatus. 
The first change toward the accomplishment of this end was effected in the substitution of two plane surfaces of larger dimensions in place of the reflecting plates formerly used. For this purpose the optical firm of Carl Zeis, of Jena, most generously provided us with two plates with plane surfaces, 4 centim. square and 1 centim. thick, one of crown glass and the other of fluorite. The plates were set in metal frames, and the distance between them regulated by a system of screws, as in the former case. With the exception of the extreme edges both plates were ground to a truly plane surface. A rectangular opening in a diaphragm, placed in the path of the rays, allowed only light to enter the slit of the spectroscope which had been reflected from the central portion of the plates. The interference-bands thus produced were unusually sharp, as can be seen from the pronounced minima of the curves in figs. 1, 2, and 3 (Plate $\mathrm{II}_{\text {.) }}$, which represent the three different energy-spectra. Hardly need it be mentioned that in the following experiments the entire optical system consisted wholly of rock-salt and fluorite.

The delicacy of the bolometer was increased chiefly by using a galvanometer of the highest degree of sensitiveness which one of us had constructed, and which will be described in detail in a forthcoming paper. The coils of the galvanometer in series measured 140 ohms resistance. When the period of the needle was reduced for the single swing to 10 seconds, one millimetre deflexion on the scale indicated a current of $1 \cdot 3 \times 10^{-11}$ ampere. With this degree of astaticism the zeropoint of the needle was perfectly constant.

The bolometer with which the following determinations were made is described as No. 2 in the previous paper. It consisted of two strips of platinum, 12 millim. long and 0.05 millim. wide, each having about $80 \mathrm{ohms}$ resistance, only one of them being exposed to radiation. With the aid of the new galvanometer we were able to trace a sensitiveness of $0.000003^{\circ} \mathrm{C}$. per millimetre deflexion. A standard candle one metre distant produced a deflexion of 400 millim.

With the exception of the changes here mentioned all pieces of apparatus were identical with those formerly described. The relative positions, moreover, of the instrument, as well as the manner and the order in which the operations were performed, were retained unchanged. We can therefore pass at once to the results of our observations.

Measurements were made upon the three materials so well known for their diathermanous properties-rock-salt, sylvite, and fluorite. 



\section{Rock-salt.}

We had at our disposal a prism of this mineral having a triangular base $3 \frac{1}{2}$ centim. on each side and $4 \frac{1}{2}$ centim. in height. Before being used the prism was freshly polished and its refracting angle redetermined. The observations with the bolometer gave the energy spectrum represented in fig. 1, Pl. II. The positions of the maxima and minima were corrected, as in the paper cited above, with the aid of the enveloping curve, whereby the points of contact of the two curves were used without further modification as the characteristic points in question. As the theory shows, this method gives a closer approximation to the quantities required than the method by construction given in the former paper. But little weight, however, is to be attached to the superiority of this modification, as both methods lead to results which are identical to the fourth decimal place.

Inasmuch as the present investigation was undertaken expressly for the purpose of extending measurements as far as possible into the infra-red, we were compelled to use a comparatively thick layer of air for reflecting the interfering beams of light, which brought the interference-bands in the spectrum very near together. It was quickly found, however, that even the narrow width of the bolometer and the impurity of the spectrum, caused by the aberration of the lenses, placed a limit beyond which the further reduction of the breadth of the interference-bands could not be carried. With the feeble dispersion of the materials used, this limit was practically reached when the visible spectrum was crossed by seven or eight interference-bands, which gave a value to the constant $K=2 d \cos i$ of about $8.5 \mu$. According to this, the minimum of the first order, which is the farthest possible attainable point in the infra-red, has a wave-length $\lambda=8.5 \mu$. Then follow the maximum of the second order and the corresponding minimum, which have wave-lengths $\lambda=5 \cdot 7 \mu$ and $\lambda=43 \mu$ respectively. Although the curvature of the curve of dispersion in this region is slight, it seems to us, nevertheless, desirable to add, for greater accuracy in our measurements, other possible points to the small number already obtained. In order to attain this end, we found it advantageous to use not only the corrected positions of the maxima and minima and their corresponding wave-lengths for plotting the curve of dispersion, but also the points of intersection of the energy curve $G=f(\alpha)$ (see fig. 1) with the curve of mean energy $\mathbf{R}=f(\alpha)$, since the wave-lengths corresponding to the abscisse of these points are easily calculated. This 
Rays of Great Wave-length in Rock-salt, Sylvite, \&c. 39

latter curve might have been observed directly, had the distance between the plates enclosing the reflecting layer of air been sufficiently increased. This curve $\mathrm{R}=f(a)$, which represents the distribution of energy when no interference is present, can be constructed, however, with sufficient accuracy, when at each point an ordinate is erected equal to the mean of the ordinates of the corresponding points of the enveloper, $\mathrm{P}$ and $\mathrm{Q}$. If the curve $\mathrm{G}=f(\alpha)$ is intersected at any point by the curve $\mathrm{R}=f(\alpha)$, then the amplitude for the abscissa of this point must have the same magnitude which it would have attained had a superposition of the energy of the two beams taken place without interference.

The vibratory motion of the two beams whose amplitude and period are $\mathrm{A}$ and $\mathrm{T}$ respectively, may be represented by the equations

$$
\begin{aligned}
& y_{1}=\mathrm{A} \sin 2 \pi\left(\frac{t}{\mathrm{~T}}-\frac{x}{\lambda}\right), \\
& y_{2}=\mathrm{A} \sin 2 \pi\left(\frac{t}{\mathrm{~T}}-\frac{x+\mathrm{K}+\frac{\lambda}{2}}{\lambda}\right) .
\end{aligned}
$$

They unite to form the ray

$$
\begin{aligned}
\mathrm{Y} & =2 \mathrm{~A} \cos \frac{\pi\left(\mathrm{K}+\frac{\lambda}{2}\right)}{\lambda} \sin 2 \pi\left(\frac{t}{\mathrm{~T}}-\frac{x+\frac{\mathrm{K}}{2}+\frac{\lambda}{4}}{\lambda}\right) \\
& =2 \mathrm{~A} \sin \frac{\pi \mathrm{K}}{\lambda} \sin 2 \pi\left(\frac{t}{\mathrm{~T}}-\frac{x+\frac{\mathrm{K}}{2}+\frac{\lambda}{4}}{\lambda}\right) .
\end{aligned}
$$

It follows from what has been said above that, for the abscissa of the point of intersection of the two curves $R$ and $G$, the amplitude of the beam $\mathrm{Y}, \mathrm{viz} .2 \mathrm{~A} \sin \frac{\pi \mathrm{K}}{\lambda}$, must equal $\pm A \sqrt{2} . \quad \lambda$ is accordingly determined from the equation

$$
\begin{gathered}
\sin \frac{\pi \mathrm{K}}{\lambda}= \pm \frac{1}{2} \sqrt{2,} \\
\frac{\pi \mathrm{K}}{\lambda}=\frac{\pi}{4}-\frac{3 \pi}{4}, \frac{5 \pi}{4} \ldots \frac{(2 n+1) \pi}{4},
\end{gathered}
$$

where $n$ is any whole number. The wave-length, therefore, of each point of intersection is given by the equation

$$
\lambda=\frac{4 \mathrm{~K}}{2 n+1} \text {. }
$$

A knowledge of the order of the adjacent maxima and minima gives at once an interpretation to the quantity $n$. If 
the point of intersection in question lies in such a way that the adjacent minimum ( $m$ th order) lies on the side of the longer wave-lengths, and the adjacent maximum on the side of the shorter wave-lengths, then $n=2 \mathrm{~m}$.

The introduction of these points in the calculation of the eurve of dispersion made it possible for us to conduct the observations with interference-bands as broad as werenecessary, and at the same time to obtain a sufficiently great number of points to enable us to ascertain the character of the curve of dispersion with nearly the same degree of accuracy as in those portions of the spectrum lying but little beyond the reach of the eye.

At this point mention should be made of a peculiarity of the energy-curve, which may be observed in the drawing (fig. 1). The deflexions of the galvanometer, at the point of the last minimum $a_{11}$, not only sink to zero, but even assume negative values. The canse of this singularity, which also appears to a smaller degree in the energy-eurve of fluorite, is to be found in the fact that the second unilluminated arm of the bolometer, which was placed in the apparatus within a casing of hard-rubber, notwithstanding this covering received upon its surface a greater amount of energy than the first arm, which was exposed to the direct radiation. The plansibility of this explanation is increased when we remember that the covered resistance is then at a portion of the spectrum in which the mean energy is 50 times greater than in the neighbourhood of the minimum $a_{11}$, and that ebonite is not opaque to thermal radiation of great wave-length.

In the following Table are found the results of the observed indices of refraction and wave-lengths. The first column, entitled "Name," gives the quality of the characteristic point in question, as Fraunhofer line, minimum $(a)$, maximum $(b)$, or point of intersection $(c)$ of the curves $G$ and $R$; the second column contains the angle of deviation $\alpha$ as measured on the graduated circle ; the third contains the index of refraction $n$, calculated from the refracting angle $\phi$ and the angle of deviation $\alpha$ according to the formula

$$
n=\frac{\sin \frac{\phi+\alpha}{2}}{\sin \frac{\phi}{2}} ;
$$

the fourth column contains finally the wave-length which is calculated from the order $m$ of the interference-band and the constant $\mathrm{K}=2 d \cos i$. The curve of dispersion plotted from the data of this Table is found in fig. 4,a. 


\section{TABLE I.}

Refracting Angle of the Rock-salt Prism, $\phi=60^{\circ} 2^{\prime}$. $\mathrm{K}=8.307 \mu ; a_{1}$ is 11th Order.

\begin{tabular}{|c|c|c|c|}
\hline Name. & $a$. & $n$. & $\lambda$. \\
\hline 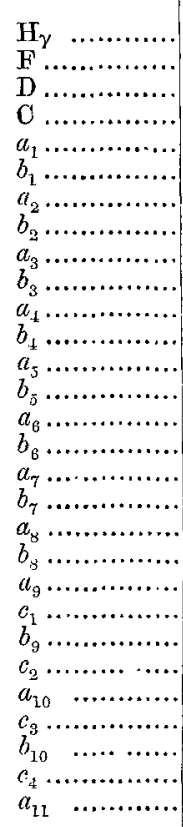 & $\begin{array}{cc}92 & 37 \\
41 & 56 \\
& 7 \\
40 & 47 \\
& 29 \\
& 22 \frac{1}{2} \\
& 16 \frac{1}{2} \\
& 11 \frac{1}{2} \\
& 7 \\
& 2 \frac{1}{2} \\
39 & 58 \\
& 54 \frac{1}{2} \\
& 51 \\
& 47 \frac{1}{2} \\
& 44 \\
& 41 \\
& 38 \\
& 35 \\
& 32 \\
& 28 \\
& 22 \frac{1}{2} \\
& 18 \\
& 12 \frac{1}{2} \\
& 7 \\
& 2 \\
38 & 56 \\
& 49 \\
& 47 \frac{1}{2} \\
& 24\end{array}$ & $\begin{array}{l}1.5607 \\
1.5531 \\
1.5411 \\
1.5404 \\
1.5370 \\
1.5358 \\
1.5347 \\
1.5337 \\
1.5329 \\
1.5321 \\
1.5313 \\
1.5305 \\
1.5299 \\
1.5293 \\
1.5286 \\
1.5280 \\
1.5275 \\
1.5270 \\
1.5264 \\
1.5257 \\
1.5247 \\
1.5239 \\
1.5230 \\
1.5217 \\
1.5208 \\
1.5197 \\
1.5181 \\
1.5163 \\
1.5138\end{array}$ & $\begin{array}{l}\mu . \\
0 \cdot 434 \\
0 \cdot 485 \\
0 \cdot 589 \\
0 \cdot 656 \\
0 \cdot 755 \\
0 \cdot 790 \\
0 \cdot 831 \\
0 \cdot 876 \\
0 \cdot 423 \\
0 \cdot 978 \\
1 \cdot 035 \\
1 \cdot 107 \\
1 \cdot 186 \\
1 \cdot 277 \\
1 \cdot 384 \\
1 \cdot 511 \\
1 \cdot 660 \\
1 \cdot 845 \\
2 \cdot 076 \\
2 \cdot 372 \\
2 \cdot 771 \\
3 \cdot 023 \\
3 \cdot 320 \\
3 \cdot 690 \\
4 \cdot 150 \\
4 \cdot 745 \\
5 \cdot 540 \\
6 \cdot 647 \\
8 \cdot 307\end{array}$ \\
\hline
\end{tabular}

It is well known that Professor Langley*, by a method wholly different from the one here described, was able to follow the dispersion in rock-salt to a wave-length $\lambda=5 \cdot 3 \mu$. $H_{\theta}$ found, in these experiments, that the curve of dispersion from about $\lambda=2 \mu$ on followed very nearly a straight line. Owing to the fact that even with the elaborate means at hand he was unable to extend measurements by his method farther than this in the direction of the long wave-lengths, he concluded to extend this straight line throughout the still more distant region of the infra-red in which his observations were taken.

Many theoretical objections are at once suggested by so extensive an exterpolation. Among these criticisms may be

$$
\text { * Ann. de Chim. et de Phys. [6] ix. p. } 433 \text { (1886). }
$$


mentioned one in particular, that, from a definite wavelength on, the indices of refraction would assume negative values, which points to an utter impossibility. There remained, however, the possibility that within the limits of Professor Langley's measurements of energy, the straight line exterpolation gave results which were at least a first approximation to the true value. A glance at the curve (fig. 4, a) shows, on the other hand, that in reality this is not the case. Indeed it is true that our own curve of dispersion tends toward a straight line until a point is reached almost as distant as $\lambda=5 \mu$; but at $\lambda=5 \mu$ the curve begins gradually to lessen its inclination to the horizontal axis of wave-lengths; and at $\lambda=8 \mu$ the effect of this curvature is so considerable that a straight line exterpolation carried from $\lambda=5 \mu$ on to this point would introduce an error in the determination of wavelength not less than $1 \mu$.

In the following Table a comparison is made befween our results and those of Professor Langley. It is to bo noticed that his curve, as far as this is plotted from his observations, agrees fairly well with our own, but that his values given by exterpolation differ widely from those observed by us, and that their difference increases as the wave-lengths become longer. There are also added here for completeness the data obtained from the previous paper. That an easier comparison may be made with Langley's figures, the wave-lengths selected increase by multiples of $\lambda_{D}=0.589 \mu$ 。

TABLE II.

\begin{tabular}{|c|c|c|c|c|c|}
\hline Wave-length. & $\begin{array}{c}n \\
\text { (Langley) }\end{array}$ & & $\begin{array}{c}n \\
\text { (Rubens). }\end{array}$ & $\begin{array}{l}n \text { (Rubens \& } \\
\text { Snow). }\end{array}$ & $\begin{array}{l}n \text { (Langley) }- \\
n \text { (Rub.\& Sn. })\end{array}$ \\
\hline 1. $\lambda_{\mathrm{D}}=\stackrel{\mu}{0} \cdot 589$ & $(15442)$ & & 1.5441 & 1.5441 & 0.0001 \\
\hline 2. $\lambda_{D}=1.158$ & 1.5301 & & 1.5300 & $1 \cdot 5301$ & 0 \\
\hline 3. $\lambda_{D}=1.767$ & 15272 & & $1 \cdot 5269$ & 1.5272 & 0 \\
\hline 4. $\lambda_{\mathrm{D}}^{\mathrm{D}}=2 \cdot 356$ & 1.5254 & 8 & 1.5253 & 1.5256 & -0.0002 \\
\hline 5. $\lambda_{\mathrm{D}}=2945$ & 1.5243 & $\stackrel{\mathscr{B}}{g}$ & 1.5241 & 1.5240 & $+0 \cdot 0003$ \\
\hline 6. $\lambda_{\mathrm{D}}=3.534$ & 1.5227 & $\stackrel{0}{0}$ & 1.5227 & $1 \cdot 5226$ & 1 \\
\hline 7. $\lambda_{D}=4.123$ & 1.5215 & & 15214 & 1.5212 & 3 \\
\hline 8. $\lambda_{D}=4.712$ & 15201 & & 1.5202 & $1 \cdot 5200$ & 1 \\
\hline 9. $\lambda_{D}=5 \cdot 301$. & 1.5186 & & 1.5189 & $1: 5188$ & -0.0002 \\
\hline 10. $\lambda_{D}=5.890$ & 1.51727 & 舁 & $\cdots$ & 1.5177 & 5 \\
\hline 11. $\lambda_{\mathrm{D}}=6.480$ & 1.5158 & $\frac{4}{8}$ & $\cdots$ & 15166 & 8 \\
\hline 12. $\lambda_{\mathrm{D}}=7070$ & $1: 5144$ & 宂 & $\cdots$ & 1.5157 & 13 \\
\hline 13. $\lambda_{D}=7.66$ & 1.5120 & $\frac{5}{\sigma}$ & $\cdots$ & 1.5148 & 19 \\
\hline 14. $\lambda_{\mathrm{D}}=8.25$ & 1.5115 & $\ddot{a}$ & $\cdots$ & 1.5138 & 23 \\
\hline
\end{tabular}


The values, therefore, attributed by Professor Langley* to the wave-lengths in that region of the spectrum lying between $\lambda=0$ and $\lambda=5 \mu$ are unáoubtedly correct. Beyond this limit, however, at least as far as $\lambda=8 \cdot 3 \mu$, the values assumed are too small, but it is not impossible that when still greater wave-lengths are reached the sign of the error may change. The results, nevertheless, of his observations remain of the greatest interest, since it will be easily possible to apply a correction to the wave-lengths as soon as the dispersion in rock-salt can be followed to sufficiently small indices of refraction.

\section{Sylvite.}

The behaviour of rock-salt is in every respect similar to that of the mineral sylvite, to which it stands in close chemical relation. There was placed at our disposal a prism of this material, 14 millim. at the base and 20 millim. in height. The surfaces of this prism were so well polished that the refracting augle could be determined to within 0.5 minute.

In fig. 2 the observed galvanometer-deflexions are plotted as functions of the angular deviation of the arm of the bolometer. From this curve is computed the table of dispersion in the manner described above. Corresponding to this is plotted the curve of dispersion (fig. $4, b$ ).

\section{TABLE III.}

Refrneting Angle of the Prism of Sylvite, $\phi=59^{\circ} 54^{\prime}$. $\mathrm{K}=8.022 \mu ; a_{1}$ is 10 th Order.

\begin{tabular}{|c|c|c|c|c|c|c|c|}
\hline Name. & $a$. & $n$ & $\lambda$. & Name. & $\alpha$. & n. & $\lambda$. \\
\hline $\mathrm{H}_{\gamma}$ & 3730 & 1.5048 & $\stackrel{\mu}{0.434}$ & $b_{x}$. & 355 & $1 \cdot 4766$ & 1.458 \\
\hline $\mathrm{F}$. & 3655 & 1.4981 & 0.486 & & & $\mathrm{l} \cdot 4761$ & $\begin{array}{l}1 \cdot 603 \\
1 \cdot 603\end{array}$ \\
\hline D .. & & 1.4900 & 0.589 & $b_{6}^{\circ}$. & $3459 \frac{1}{2}$ & $1 \cdot 4755$ & 1.781 \\
\hline C. & 3557 & $1 \cdot 4868$ & 0.656 & & $56 \frac{2}{2}$ & $1 \cdot 4749$ & 2005 \\
\hline$a_{1}$. & 37 & 1.4829 & 0.802 & $b_{7} \ldots \ldots$ & 53 & 1.4742 & 2291 \\
\hline$b_{1}$. & 32 & 1.4819 & 0.845 & $a_{8} \ldots \ldots$ & 48 & 1.4732 & $2 \cdot 673$ \\
\hline$a_{2}$. & 27 & 1.4809 & 0.893 & $b_{8} \ldots \ldots$ & 43 & 1.4722 & 3209 \\
\hline$b_{3}$. & $23 \frac{1}{2}$ & $1.480 \%$ & 0944 & $c_{1} \ldots \ldots$ & $40 \frac{7}{2}$ & $1 \cdot 4717$ & 3.561 \\
\hline$a_{3}$. & 20 & $1 \cdot 4795$ & 1.003 & $a_{0}$. & 38 & 1.4712 & 4.011 \\
\hline$b_{3}$. & $16 \frac{1}{2}$ & 1.4789 & $1 \cdot 070$ & $c_{2}$. & $35 \frac{1}{2}$ & 1.4708 & 4.577 \\
\hline$a_{4}$ & 13 & $1 \cdot 4782$ & $1 \cdot 145$ & $b_{9} \ldots \ldots$ & 32 & 1.4701 & $5 \cdot 345$ \\
\hline & 10 & $1 \cdot 4776$ & 1.234 & $c_{3} \ldots \ldots$ & 28 & l.4693 & 6.412 \\
\hline$a_{\tilde{b}}$. & $7 \frac{1}{2}$ & 1.4771 & $1 \cdot 337$ & $a_{10} \quad \ldots$ & 22 & $1 \cdot 4681$ & 8.022 \\
\hline
\end{tabular}

* Langley, Sill. Journ. [3] xxxi. pp. 1-22, 1886; further [3] xxxii. pp. $83-106$, 1886, and [8] xxxviii. pp. 421-440; Phil. Mag. xxvi. p. 505, 1888. The sarne is true of the paper of Angström, Ofversigt af Kongl. Vet.-A7ad. Förhandl. ix. p. 549, 1889, and vii. p. 331, 1890, and W. H. Julius, Arch. Néerl, i, pp. 310-384, 1888. 
A study of this curve shows that the dispersion in sylvite, which in the visible spectrum is only slightly inferior to that in rock-salt, decreases in a similar manner but far more rapidly than in the latter mineral, so that at wave-length $\lambda=8 \mu$ the dispersion is only about one third of the corresponding dispersion in rock-salt.

Notwithstanding the great durability of this material, and its permanence in moist air as well as its almost perfect transparency to thermal radiations, the exceedingly rapid decrease in the dispersive power of sylvite renders this substance not so well adapted for experiments involving the use of prisms as rock-salt, whose surfaces are only with difficulty kept perfect. In the construction of condensing lenses this difficulty does not occur.

\section{Fluorite.}

The prism here examined is the same one which was used in the former investigation. The value of the refracting angle was redetermined and was found to agree very closely with the observations previously made.

For a long time we tried in vain to measure beyond wavelength $\lambda=3 \cdot 5 \mu$, the energy-spectrum produced by the fluorite prism. The results of the previous observations show the cause of our failure to be due to the fact that, after a region of comparatively feeble dispersion, the dispersive power of fluorite increases and the energy in this portion of the spectrum becomes proportionally weaker. In order to make further advances, we were finally compelled to open wider the slit of the spectrometer at those places where the radiant energy sank below a measurable quantity. The repetition of this device enabled us to reach a wave-length in the infra-red greater than $\lambda=8 \mu$. In the curve shown in fig. 3 , which represents the observed distribution of energy produced by the fluorite prism, the slit was twice opened; the first time from 0.1 millim. to 0.4 millim. when the arm of the bolometer was at a deviation $a=30^{\circ} 10^{\prime}$, and a second time from 0.4 millim. to $1 \cdot 0$ millim. at an angle of deviation $\alpha=28^{\circ} 50^{\prime}$. By this means the deflexions of the galvanometer were increased fourfold and tenfold respectively. Owing to the greatly increased dispersion and the corresponding increase in the breadth of the interference-bands, this change in the width of the slit did not materially interfere with the sharpness of the bands in this region of the spectrum. Inasmuch as only one side of the slit was movable, a correction had to be applied to the realing of the arm of the bolometer when the slit was opened.

The distribution of energy, as shown in fig. 3 , gives a curve whose character is wholly different from the representation of 
Rays of Great Wave-length in Rock-salt, Sylvite, \&c. 45

the energy-spectra produced by rock-salt or sylvite, given in figs. 1 and 2. While in these latter the breadth of the interference-bands increases only slowly as the extreme infrared is reached, amounting finally to hardly more than double the smallest value, the breadth of these bands varies in the energy-spectrum, as given by the fluorite prism, from 5 minutes to more than $2 \frac{1}{2}$ degrees. Corresponding to this peculiar characteristic in the energy-spectrum of fluorite, the quality of the dispersion in this mineral is quite different from that of the materials previously considered.

In the following Table, which contains these data, the indices of refraction are given only to three decimal places. As a result of the very considerable breadth of the interferencebands, it is impossible to locate the position of the characteristic points with the precision attainable in other cases.

Table IV.

Refracting Angle of the Fluorite Prism, $\phi=59^{\circ} 59 \frac{1}{2}^{\prime}$. $\mathrm{K}=8.070 \mu ; a_{1}$ is 10 th Order.

\begin{tabular}{|c|c|c|c|c|c|c|c|}
\hline Name. & $a$. & $n$. & $\lambda$. & Name. & a. & $n$. & $\lambda$. \\
\hline $\begin{array}{cc}\mathrm{H}_{\gamma} & \ldots \\
\mathrm{F} & \ldots \ldots \\
\mathrm{D} & \ldots \ldots \\
\mathrm{O} & \ldots \ldots \\
a_{1} & \ldots \ldots \\
b_{2} & \ldots \ldots \\
a_{2} & \ldots \ldots \\
b_{2} & \ldots \ldots \\
a_{3} & \ldots \ldots \\
b_{3} & \ldots \ldots \\
a_{1} & \ldots \ldots \\
b_{4} & \ldots \ldots \\
a_{5} & \ldots \ldots\end{array}$ & \begin{tabular}{rr}
30 & \multicolumn{1}{c}{} \\
31 & 52 \\
36 \\
29 \\
29 \\
19 \\
17 \\
141 \\
12 \\
12 \\
10 \\
8 \\
6 \\
6 \\
4 \\
2
\end{tabular} & $\begin{array}{l}1.4398 \\
1.4372 \\
1.4340 \\
1.4395 \\
1.4307 \\
1.4303 \\
1.4299 \\
1.4294 \\
1.4290 \\
1.4286 \\
1.4281 \\
1.4277 \\
1.4272\end{array}$ & $\begin{array}{l}\mu \\
0.434 \\
0.485 \\
0.589 \\
0.656 \\
0.807 \\
0.850 \\
0.896 \\
0.950 \\
1.009 \\
1.076 \\
1.152 \\
1.240 \\
1.345\end{array}$ & 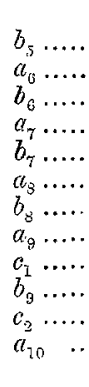 & $\begin{array}{rr}30 & 59 \\
55 \frac{1}{2} \\
51 \\
51 \\
46 \\
38 \\
29 \\
29 \\
13 \\
2946 \\
29 \\
4 \\
2830 \\
27 & 5\end{array}$ & $\begin{array}{l}1.4267 \\
1.4260 \\
1.4250 \\
1.4240 \\
1.4324 \\
1.4205 \\
1.4174 \\
1.4117 \\
1.408 \\
1.403 \\
1.396 \\
1.378\end{array}$ & $\begin{array}{l}\mu \\
1 \cdot 466 \\
1 \cdot 613 \\
1 \cdot 702 \\
2 \cdot 019 \\
2 \cdot 303 \\
2 \cdot 689 \\
3 \cdot 292 \\
4.035 \\
4 \cdot 62 \\
5 \cdot 38 \\
6 \cdot 46 \\
8.07\end{array}$ \\
\hline
\end{tabular}

The curve of dispersion, shown in fig. 4, c, exhibits more graphically than this table the peculiar character of the dispersion. From this it is seen that the dispersive power of fluorite decreases as far as $\lambda=2 \mu$ and then gradually increases, reaching at $\lambda=8 \mu$ a value only slightly inferior to the value of the dispersion in the red.

Compared with rock-salt and sylvite, the dispersion of fluorite in the visible spectium is exceedingly small and unusually great in the infra-red; so that this material is pcculiarjy well adapted to the production of prismatic heatspectra, an advantage which is still further increased by the ease with which it can be worked and by the permanence of its surface in the air.

Physical Laboratory, University of Berlin, June 1892. 